\title{
Conference Paper Study of Rheological Properties of Industrial Lubricants
}

\author{
Ajay Vasishth, ${ }^{1}$ Piyush Kuchhal, ${ }^{2}$ and Gagan Anand ${ }^{2}$ \\ ${ }^{1}$ Department of Physics, Chandigarh University, Chandigarh, Punjab 160017, India \\ ${ }^{2}$ Department of Physics, University of Petroleum and Energy Studies, Dehradun 248007, India
}

Correspondence should be addressed to Gagan Anand; gagan4567@rediffmail.com

Received 11 February 2014; Accepted 11 March 2014; Published 14 May 2014

Academic Editors: R. K. Shivpuri and G. N. Tiwari

This Conference Paper is based on a presentation given by Gagan Anand at "National Conference on Advances in Materials Science for Energy Applications" held from 9 January 2014 to 10 January 2014 in Dehradun, India.

Copyright (C) 2014 Ajay Vasishth et al. This is an open access article distributed under the Creative Commons Attribution License, which permits unrestricted use, distribution, and reproduction in any medium, provided the original work is properly cited.

The most important rheological parameter for lubricants is viscosity as it also affects the tribological properties like friction between interacting surfaces and wear. This research intends to study the relationship between viscosity and temperature at different shear rates for multiple grades of three different categories of lubricants used for different applications viz. L1: MG20W50 (engine oil), L2: SAE20W50 (engine oil), L3: MC20W50 (mineral engine oil), L4: EP90 (gear oil), and L5: DXTIII (steering fluid). Constant high dynamic viscosity, shear stress, and low compressibility at different temperatures in multigrade as well as single grade industrial oil will help to maintain the surface film over the period of time and hence the reduction in wear. The dynamic viscosity of these chosen samples has been measured experimentally in temperature range of 20 to $50^{\circ} \mathrm{C}$. The measurements have been extended to observe the dependence of shear rate, time, and temperature on the dynamic viscosity. All the samples are observed to behave like Newtonian fluids in the entire temperature range of study. Further, all samples seem to obey the Arrhenius relationship with temperature. Shear stress shows linear variation with shear rate exhibiting uniform viscosity which is substantiated by almost no variation in dynamic viscosity with shear rate for value above 5 per second.

\section{Introduction}

The role of lubrication is an important part in the field of tribology. Lubrication is to smoothen the movement of one surface over another and to maintain the viscoelastic behavior [1].

Lubricants are commonly used for lubrication to reduce the friction and wear of surfaces in contact [2] and effective heat transfer due to good thermal conductivity. Most lubricants are liquids (such as mineral oils, synthetic oil, silicon fluids, water, etc.). Selection of lubricant is very important for providing machine tools a longer life. To select an appropriate lubricant, it is necessary to know its properties, lubrication system of applied machinery, conditions of machinery, cost of lubricant [3]. Common properties of lubricating oil are: viscosity, viscosity index, density, compressibility, surface tension, cloud point, pour point or low temperature property, flash point, friction coefficient, high boiling point, low freezing point, thermal stability, corrosion prevention, high resistance to oxidation, and so forth. The most important property is its viscosity. Viscosity is a function of temperature and pressure. The relationship between the viscosity and temperature and the relationship between viscosities and pressure are also important in lubricant rheology as well as for the life of machine elements. Just as temperature rise reduces the viscosity of lubricating oil, also an increase in pressure produces a rise in its viscosity. Barus expressed a relation between viscosity and pressure introducing a constant $\alpha$ named pressure-viscosity coefficient [4]. In recent years, pressure viscosity relationship became an important parameter of lubricating oil to understand its performance, especially in high-temperature applications, so viscosity measurement becomes an important tool for doing so. Rheology is the study of the flow of matter: mainly liquids but also soft solids or solids under conditions in which they flow rather than deform elastically. It would be difficult to imagine any 
TABLE 1: Shear stress, dynamic viscosity and torque versus temperature measurements for the samples L1, L2, L3, L4, L5 at shear rate 10 .

\begin{tabular}{lccccccccccccccc}
\hline $\begin{array}{l}\text { Temp } \\
\left({ }^{\circ} \mathrm{C}\right)\end{array}$ & L1 & L2 & L3 & L4 & L5 & L1 & L2 & L3 & L4 & L5 & L1 & L2 & L3 & L4 & L5 \\
\hline 20 & 0.45 & 0.61 & 0.46 & 0.51 & 0.24 & 4.51 & 6.07 & 4.58 & 5.09 & 2.43 & 0.06 & 0.08 & 0.06 & 0.07 & 0.03 \\
25 & 0.38 & 0.49 & 0.36 & 0.44 & 0.19 & 3.84 & 4.91 & 3.64 & 4.44 & 1.93 & 0.05 & 0.06 & 0.05 & 0.06 & 0.03 \\
30 & 0.29 & 0.40 & 0.34 & 0.37 & 0.15 & 2.87 & 4.04 & 3.40 & 3.69 & 1.46 & 0.04 & 0.05 & 0.04 & 0.05 & 0.02 \\
35 & 0.26 & 0.34 & 0.27 & 0.30 & 0.12 & 2.64 & 3.40 & 2.68 & 3.03 & 1.17 & 0.03 & 0.04 & 0.03 & 0.04 & 0.02 \\
40 & 0.24 & 0.29 & 0.17 & 0.27 & 0.12 & 2.37 & 2.90 & 1.68 & 2.69 & 1.19 & 0.03 & 0.04 & 0.02 & 0.04 & 0.02 \\
45 & 0.24 & 0.25 & 0.16 & 0.24 & 0.07 & 2.37 & 2.49 & 1.59 & 2.39 & 0.71 & 0.03 & 0.03 & 0.02 & 0.03 & 0.01 \\
50 & 0.22 & 0.20 & 0.15 & 0.23 & 0.06 & 2.22 & 1.95 & 1.52 & 2.26 & 0.59 & 0.03 & 0.03 & 0.02 & 0.03 & 0.01 \\
\hline
\end{tabular}

TABLE 2: Shear stress, viscosity and torque measurements for the samples L1, L2, L3, L4 and L5 at room temperature.

\begin{tabular}{|c|c|c|c|c|c|c|c|c|c|c|c|c|c|c|c|}
\hline \multirow{2}{*}{$\begin{array}{l}\text { Shear rate } \\
(1 / s]\end{array}$} & \multicolumn{5}{|c|}{ Viscocity } & \multicolumn{5}{|c|}{ Stress } & \multicolumn{5}{|c|}{ Torque } \\
\hline & $\mathrm{L} 1$ & L2 & $\begin{array}{c}\mathrm{L} 3 \\
(\mathrm{~Pa} \cdot \mathrm{s})\end{array}$ & $\mathrm{L} 4$ & L5 & $\mathrm{L} 1$ & $\mathrm{~L} 2$ & $\begin{array}{r}\mathrm{L} 3 \\
(\mathrm{~Pa})\end{array}$ & L4 & L5 & $\mathrm{L} 1$ & L2 & $\begin{array}{c}\mathrm{L} 3 \\
(\mathrm{mNm})\end{array}$ & L4 & L5 \\
\hline 10 & 0.41 & 0.53 & 0.56 & 0.47 & 0.20 & 4.06 & 5.33 & 5.60 & 4.67 & 1.98 & 0.05 & 0.07 & 0.07 & 0.06 & 0.02 \\
\hline 20 & 0.37 & 0.50 & 0.42 & 0.43 & 0.18 & 7.41 & 10.10 & 8.47 & 8.51 & 1.78 & 0.10 & 0.13 & 0.11 & 0.11 & 0.03 \\
\hline 30 & 0.35 & 0.48 & 0.40 & 0.41 & 0.13 & 10.40 & 14.50 & 11.90 & 12.20 & 1.27 & 0.14 & 0.19 & 0.15 & 0.16 & 0.04 \\
\hline 40 & 0.34 & 0.47 & 0.39 & 0.40 & 0.11 & 13.60 & 18.70 & 15.70 & 15.80 & 1.12 & 0.18 & 0.24 & 0.21 & 0.21 & 0.05 \\
\hline 50 & 0.33 & 0.46 & 0.39 & 0.39 & 0.11 & 16.70 & 22.90 & 19.40 & 19.40 & 1.05 & 0.22 & 0.30 & 0.25 & 0.25 & 0.06 \\
\hline 60 & 0.33 & 0.45 & 0.39 & 0.38 & 0.10 & 19.80 & 27.30 & 23.20 & 22.90 & 1.02 & 0.26 & 0.35 & 0.30 & 0.30 & 0.07 \\
\hline 70 & 0.33 & 0.45 & 0.39 & 0.38 & 0.10 & 23.00 & 31.70 & 27.00 & 26.50 & 1.00 & 0.30 & 0.41 & 0.35 & 0.34 & 0.09 \\
\hline 80 & 0.33 & 0.45 & 0.39 & 0.38 & 0.10 & 26.50 & 36.00 & 30.80 & 30.30 & 1.01 & 0.34 & 0.47 & 0.40 & 0.39 & 0.10 \\
\hline 90 & 0.33 & 0.45 & 0.39 & 0.38 & 0.10 & 29.40 & 40.40 & 34.70 & 34.00 & 0.99 & 0.38 & 0.53 & 0.45 & 0.44 & 0.12 \\
\hline 100 & 0.33 & 0.45 & 0.39 & 0.38 & 0.10 & 32.70 & 44.80 & 38.70 & 37.90 & 0.98 & 0.42 & 0.58 & 0.50 & 0.49 & 0.13 \\
\hline
\end{tabular}

type of machinery without lubrication. One of the single largest applications for lubricants, in the form of motor oil, is protecting the internal combustion engines in motor vehicles and powered equipment. Typically lubricants contain $90 \%$ base oil (most often petroleum fractions, called mineral oils) and less than $10 \%$ additives. Vegetable oils or synthetic liquids such as hydrogenated polyolefin, esters, silicones, fluorocarbons, and many others are sometimes used as base oils. Additives deliver reduced friction and wear, increased viscosity, improved viscosity index, resistance to corrosion and oxidation, aging or contamination, and so forth. Lubricants such as 2-cycle oil are added to fuels like gasoline which has low lubricity [5]. Motor oils are derived from petroleum-based and non-petroleumsynthesized chemical compounds. Motor oils are mainly blended by using base oils composed of hydrocarbons, polyalphaolefins (PAO), and polyinternal olefins (PIO) $[6,7]$, which are organic compounds consisting entirely of carbon and hydrogen. The base oils of some high-performance motor oils however contain up to $20 \%$ by weight of esters. Lubricating oil creates a separating film between surfaces of adjacent moving parts to minimize direct contact between them, decreasing heat caused by friction and reducing wear, thus protecting the engine $[8,9]$. Maintenance of high pressure viscosity index at different temperatures in multigrade as well as single grade engine oil is key to its sustained performance. It is also a deciding factor of wear of machine parts. Better rheological behavior with temperature will lead to better tribology [10]. Mia and Ohno [11] measured adiabatic bulk modulus $K$ using Sing around technique [12]. Ohno and Hirano [13] calculated tangent bulk modulus $K_{T}$ using highpressure densitometer. Tsubouchi and Shinoda used tangential bulk modulus to characterize oily high bulk modulus fluid [14]. Prashant Thapliyal et al. [15] calculated adiabatic bulk modulus of piezoviscous elastic engine oils by measuring ultrasonic velocity using Nanofluid Interferometer.

\section{Experimental}

Industrial lubricating oils L1, L2, L3, L4, and L5 are considered as sample oils as shown in Figure 1. Viscosity with rise in temperature was measured using Rheometer MCR302 (Figure 2), SN000000, ID 80963516 from Anton Paar Gurgaon. Measuring system PP25/PE-SN25125 $(d=$ $0.4 \mathrm{~mm}$ ) with accessory TUI = P-PTD200-SN 81183777 was used.

2.1. Measurement of Viscosity, Shear Stress, and Torque with Temperature. The setup shown in Figure 2 is used to measure the shear stress $(\sigma)$, viscosity $(\eta)$, and torque $(\tau)$ for all five samples as a function of temperature in a range from $20^{\circ} \mathrm{C}$ to $50^{\circ} \mathrm{C}$ at constant shear rate $(\gamma)$ of $10 \mathrm{sec}^{-1}$. 31 data points were selected with measuring duration of $2 \mathrm{~min}$. Shear stress, viscosity, and shear rate are related to the following relation 


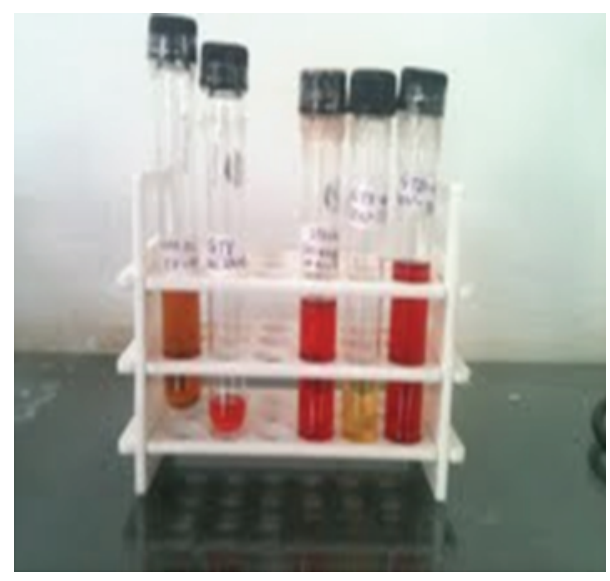

Figure 1: Samples L1, L2, L3, L4, and L5.

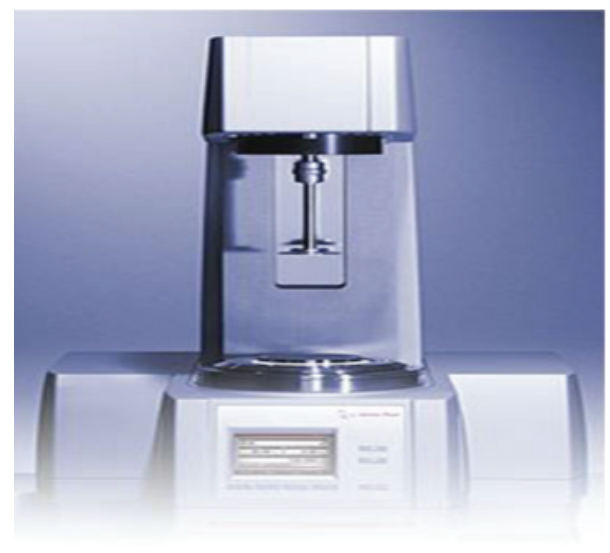

Figure 2: Anton Paar MCR302.

$\sigma=\eta \gamma$. The measured values of shear stress, viscosity, and torque with temperature are reported in Table 1.

\subsection{Measurement of Viscosity, Shear Stress, Speed, and Torque} with Shear Rate. The experimental set up mentioned in 2.0 was used for this purpose. 100 measure points were selected with duration of $2 \mathrm{~s}$ and shear rate varied from 1 to $100 / \mathrm{s}$. All the measurements were done at room temperature with variation in speed while the duration of measurement was fixed at $2 \mathrm{~s}$. The results are reported in Table 2 .

\section{Results and Discussion}

Figure 3 shows the exponential variation between dynamic viscosity with temperature for the samples L1, L2, L3, and L5, having trend for $\mathrm{L} 1$ as $\eta=1.34 e^{0.694 T}$, for $\mathrm{L} 2 \eta=1.43 e^{0.549 T}$, for L3 $\eta=1.78 e^{0.0835 T}$, for L4 $\eta=0.0991 e^{0.041 T}$, and for L5 $\eta=0.649 e^{0.0496 T}$. Here $\eta$ denotes dynamic viscosity while $T$ denotes temperature. All the samples are found to obey Reynolds' equation [16] as follows:

$$
\mu_{0}=b e^{-a T_{A}}
$$

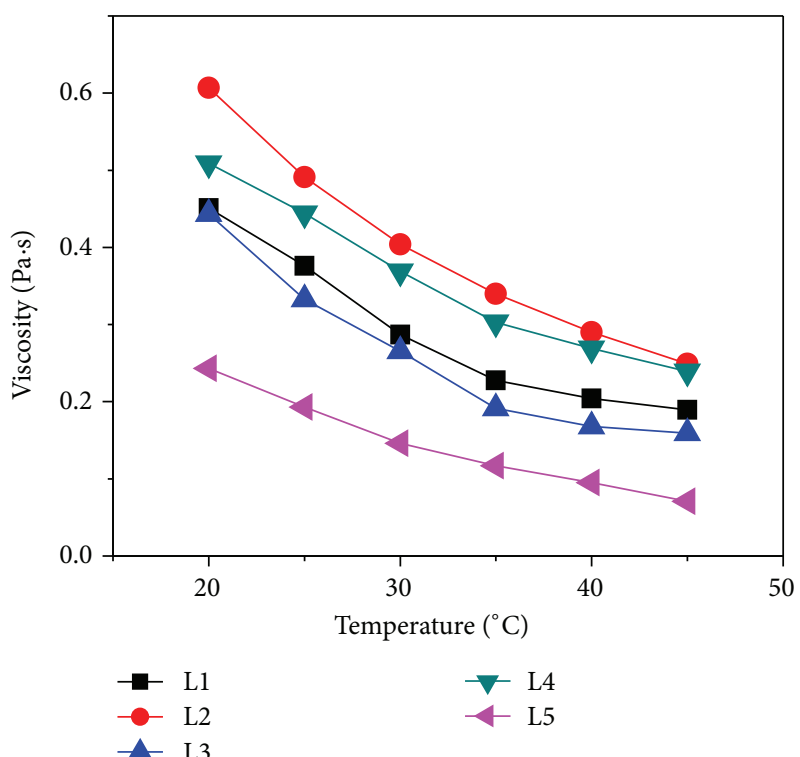

Figure 3: Dynamic viscosity versus temperature for L1, L2, L3, L4, and L5.

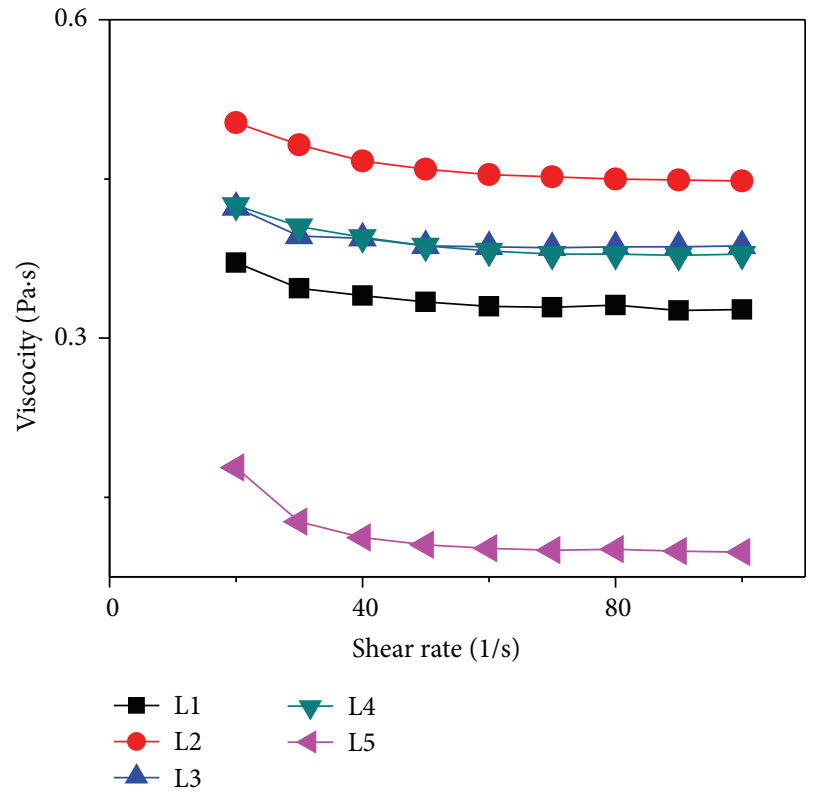

FIGURE 4: Dynamic viscosity versus shear rate for L1, L2, L3, L4, and L5.

where $\mu_{0}$ is dynamic viscosity at atmospheric pressure and $T_{A}$ is absolute temperature. Figure 4 shows the variation of viscosity with shear rate for L1, L2, L3, L4, and L5 samples. Figure 5 shows the stress versus shear rate variation which is found to be liner for all samples with L5 depicting lowest slope. Straight line graphs of stress versus shear rate depict trends for L1 as $\sigma=0.317 \gamma+0.919$, for $\mathrm{L} 2$ as $\sigma=0.433 \gamma+1.38$, for L3 as $\sigma=0.378 \gamma+0.606$, for L4 as $\sigma=0.365 \gamma+1.15$, and for L5 as $\sigma=0.099 \gamma-0.183$. Dynamic viscosity initially decreases with shear rate denoting piezoviscous behavior while for range above 10 per second it is almost constantly showing 


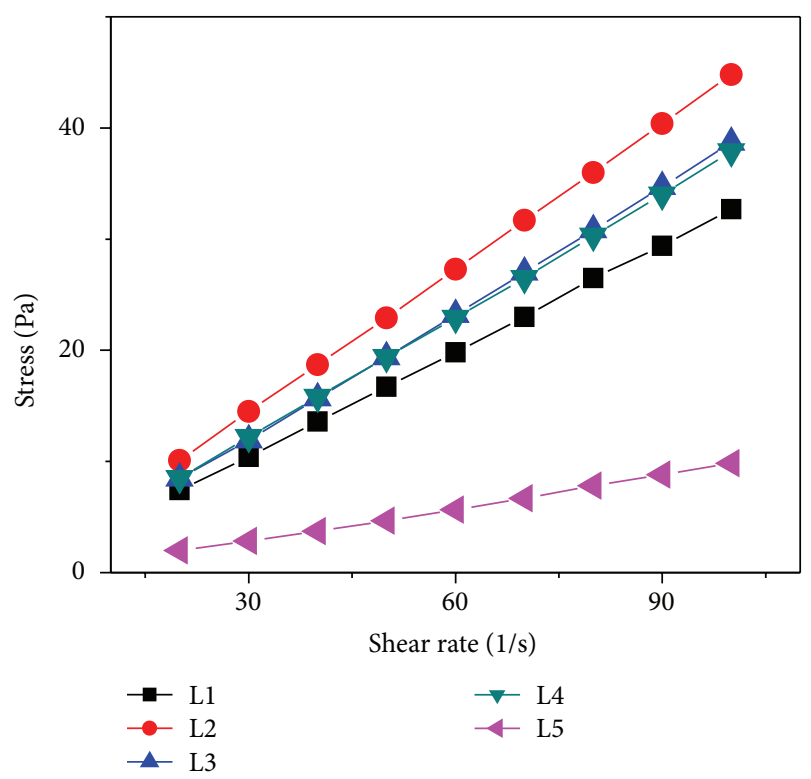

FIGURE 5: Shear stress versus shear rate for L1, L2, L3, L4, and L5.

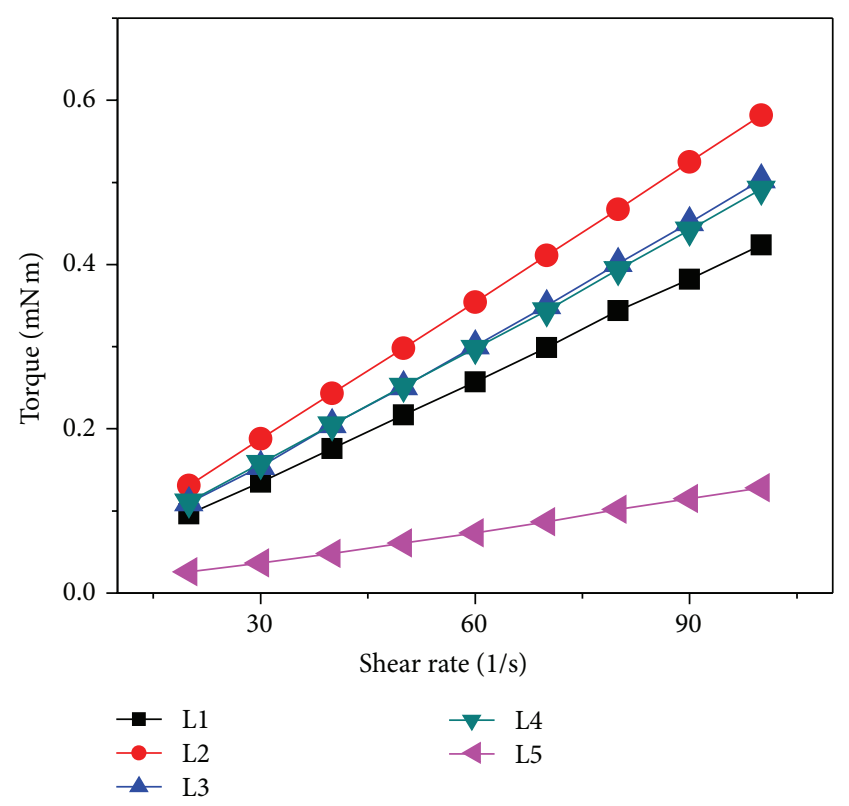

Figure 6: Torque versus shear rate for L1, L2, L3, L4, and L5.

Newtonian behavior. Figure 6 shows torque versus shear rate for all samples with almost linear dependence. The observed trends for L1 as $\tau=0.004 \gamma+0.011$, for L2 as $\tau=0.005 \gamma+0.017$, for L3 as $\tau=0.007 \gamma+0.004$, for L4 as $\tau=0.44 \gamma+0.014$, and for L5 as $\tau=0.0013 \gamma-0.002$, respectively.

\section{Conclusions}

The conclusions can be drawn as follows.

(a) L2 and L3 show relatively lower variation of dynamic viscosity with temperature, thus depicting consistent behavior with temperature variations. (b) Lowest slope of stress versus shear rate curve for L5 suggests more fluidity which is verified by its comparatively lower dynamic viscosity.

(c) Dynamic viscosity decreases with temperature for all the samples showing second degree polynomial variation.

(d) Shear stress shows linear variation with shear rate showing uniformity in viscosity which is substantiated by almost no variation in dynamic viscosity with shear rate for value above 5 per second. It shows that the given lubricants depict Newtonian behavior in hydrostatic regime.

(e) Lubricants L1, L2, L3, and L5 obey Reynolds' equation $\mu_{0}=b e^{-a T_{A}}$.

\section{Conflict of Interests}

The authors declare that there is no conflict of interests regarding the publication of this paper.

\section{References}

[1] B. Bhushan, Principles and Applications of Tribology, John Wiley \& Sons, New York, NY, USA, 1999.

[2] A. R. Lansdown, Lubrication: A Practical Guide to Lubricant Selection, Pergamon Press, Oxford, UK, 1982.

[3] W. R. Schowalter, Mechanics of Non-Newtonian Fluids, Pergamon Press, Oxford, UK, 1978.

[4] C. Barus, "Isothermal, isopiestics and isometrics relative to viscosity," The American Journal of Science, vol. 45, pp. 87-96, 1893.

[5] Engine Oil Licensing and Certification System, API 1509, 15th edition, 2002, Appendix E: API Base Oil Interchangeability Guidelines for Passenger Car Motor Oils and Diesel Engine Oils (revised).

[6] R. H. Schlosberg, J. W. Chu, G. A. Knudsen, E. N. Suciu, and H. S. Aldrich, "High stability esters for synthetic lubricant applications," Lubrication Engineering, vol. 57, no. 2, pp. 21-26, 2001.

[7] M. Woydt, "No/Low SAP and alternative engine oil development and testing," Journal of ASTM International, vol. 4, no. 10, 2007.

[8] K. Dieter, Lubricants and Related Products, Chemie, Hoboken, NJ, USA, 1984

[9] N. Ohno, N. Kuwano, and F. Hirano, "Observation of mechanical behaviour of solidified oils by using photoelastic method," Journal of Japan Society of Lubrication Engineers, vol. 33, no. 9, pp. 693-699, 1988.

[10] S. Mia and N. Ohno, "Relation between low temperature fluidity and sound velocity of lubricating oil," Tribology International, vol. 43, no. 5-6, pp. 1043-1047, 2010.

[11] S. Mia and N. Ohno, "Prediction of pressure-viscosity coeffi cient of lubricating oils based on sound velocity," Lubrication Science, vol. 21, no. 9, pp. 343-354, 2009.

[12] S. Mia and N. Ohno, "Effect of high adiabatic bulk modulus on the rheological behavior of hydraulic fluid," in Proceedings of the 13th Asian Congress of Fluid Mechanics, pp. 17-21, Dhaka, Bangladesh, December 2010. 
[13] N. Ohno and F. Hirano, "High pressure rheology analysis of traction oils based on free volume measurements," Lubrication Engineering, vol. 57, no. 7, pp. 16-22, 2001.

[14] T. Tsubouchi and J. Shinoda, "Characterization of oily high bulk modulus fluid," in Proceedings of the World Tribology Congress, p. 532, Kyoto, Japan, September 2009.

[15] P. Thapliyal, R. Sethi, A. Vasishth, and G. Anand, "Study of elastic and rheological properties of piezoviscous elastic engine oils," in Proceedings of the International Conference on Nanotechnology in Conventional and Alternative Energy Systems: A Global Status and Pathways, University of Petroleum and Energy Studies, Dehradun, India, August 2013.

[16] D. Knezevic and V. Savic, "Mathematical modelling of changing of dynamic viscosity, as a function of temperature and pressure, of mineral oils for hydraulic systems," Facta Universitatis, Series: Mechanical Engineering, vol. 4, no. 1, pp. 27-34, 2006. 

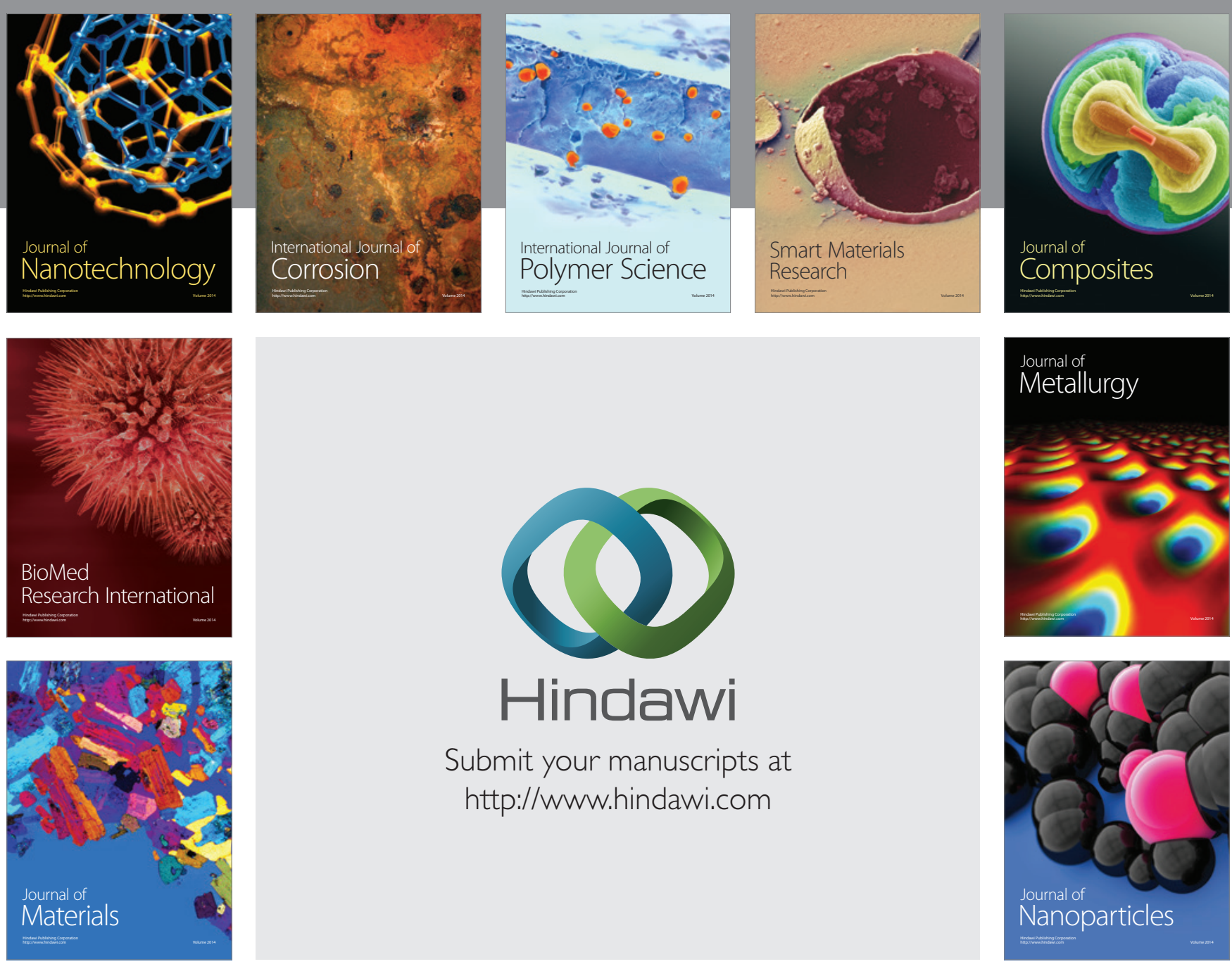

Submit your manuscripts at http://www.hindawi.com
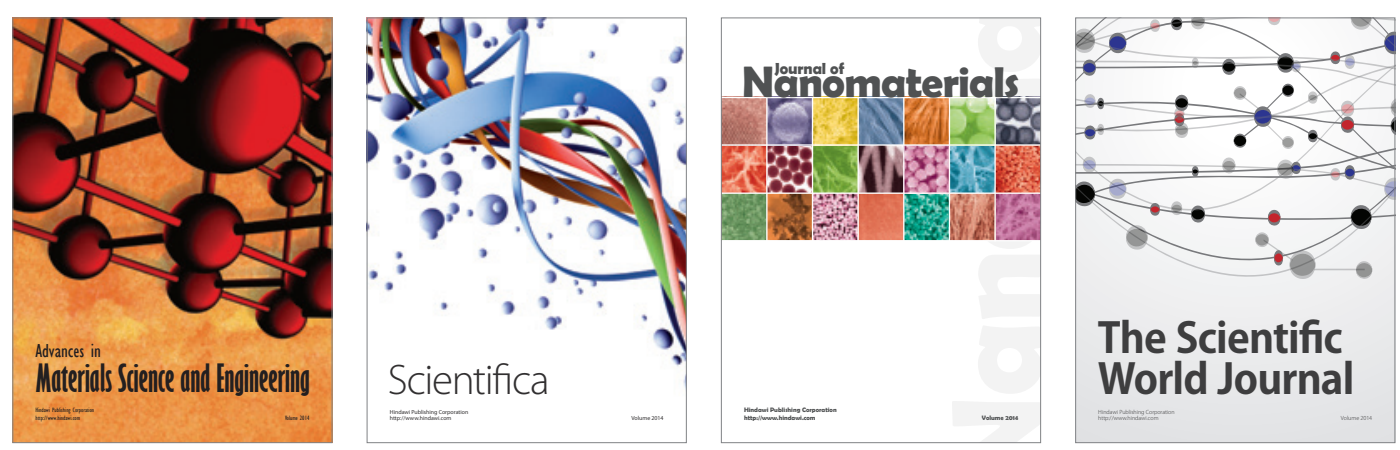

\section{The Scientific World Journal}
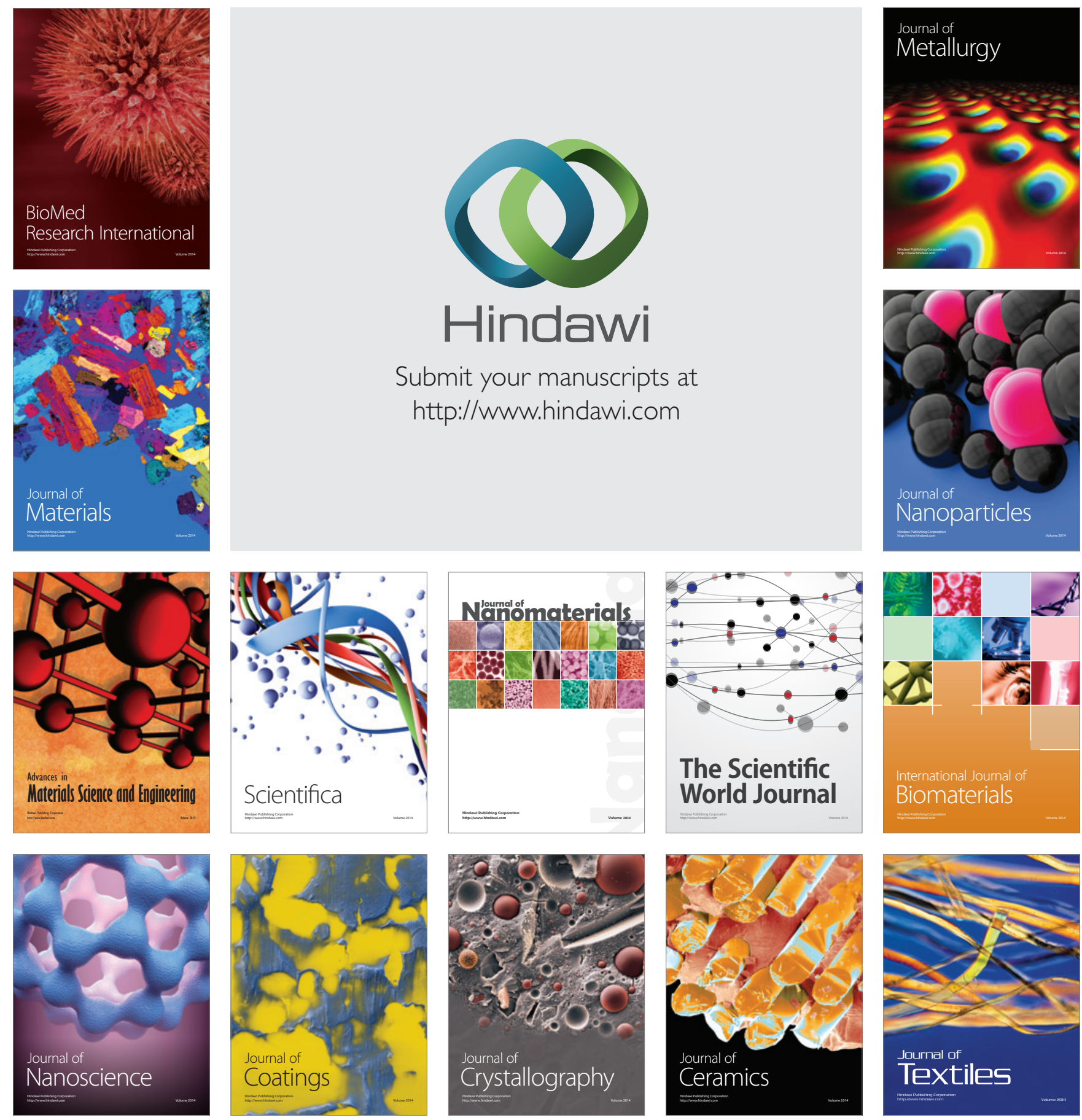